\title{
Interés de la histeroscopia diagnóstica en la evaluación de la infertilidad femenina
}

\author{
Christian Pérez-Villabona; Daniel Cohen; Philippe Roge; Geraldine Porcu; Leon Boubli*
}

\section{RESUMEN}

OBJETIVO: Evaluar la importancia de la histeroscopia diagnóstica en el contexto del estudio de la infertilidad.

DISEÑO METODOLOGICO: Estudio retrospectivo, descriptivo sobre 146 pacientes que consultaron al centro, por problemas de infertilidad, a las que se les practicó una histeroscopia diagnostica dentro de su estudio; entre Enero de 1993 y Julio de 1996, en el CENTRE DE PROCREATIONS MEDICALEMENT ASSISTEES, SERVICE DE GYNECOLOGIE C., HÔPITAL DE LA CONCEPTION. MARSEILLE-FRANCE.

RESULTADOS: La frecuencia de las principales lesiones encontradas al examen endoscópico fue:-anomalías del canal endocervical: $8 \%$, -polipos: $11 \%$, -fibromas: $2-4 \%$, sinequias: $36 \%$,-malformaciones uterinas $10 \%$, de estas últimas $54 \%$ correspondieron a tabiques uterinos. Ninguna de las lesiones del canal endocervical encontradas a la endoscopia, fue evidenciada por la histerosalpingografía. Cuando se comparó el resultado del examen endoscópico y radiográfico, la concordancia fue del $47 \%$. Dentro del grupo de pacientes que tenían antecedentes de Procreación Médica Asistida (PMA) v. gr. FIV, ICSI; $96 \%$ tuvieron anomalías a la histeroscopia; y entre las pacientes con antecedente de varios intentos fallidos de PMA, el porcentaje de exámenes anormales aumentó proporcionalmente al número de intentos fallidos de PMA.

CONCLUSIONES: -La frecuencia de las lesiones intracavitarias diagnosticadas por endoscopia, en las pacientes infértiles, es diferente a la de la población general. -La histeroscopia es el examen de elección en anomalías del canal endocervical. -La exploración endoscópica de la cavidad uterina debe recomendarse en el estudio de la infertilidad, como complemento de la evaluación de la permeabilidad tubárica por histerosalpingografía; y debe hacerse sistemáticamente antes de iniciar todo intento de PMA "invasivo" (que implique transferencia de embriones o de espermatozoides.)

PALABRAS CLAVES: Histeroscopia, infertilidad, estudio mujeres.

\section{SUMMARY}

OBJETIVE: To evaluate the importance of diagnostical hysteroscopy in the context of infertility studies.

METHODS AND MATERIALS: Retrospective and descriptive study about 146 patients with infertility problems whom were practiced diagnostical hysteroscopy between january 1993 and july 1996 at the "Centre de Procreations Medicallement Assistees, Service de Gynecologie, Hospital De La Conception". Marselle - France.

RESULTS: The frecuency of principal lesions found by endoscopy were endocervical canal anomalities $8 \%$, polypus $11 \%$, Fibroma $2.4 \%$, synechiae $36 \%$, uterus malformations $11 \%$. None of the endocervical canal lesions found by endoscopy was evidence by hysterosalpingography. The concordance between the endoscopy and radygraphye exam was $47 \%$. In the group of patients that had previous Medical Assisted Procreations (MAP) v.gr. IVP, ICSI, $96 \%$ had anomalies at hysteroscopy; and in the patients with previous faulis of MAP, the percentage anormal exams increased proportionally to the faults attempted.

CONCLUSIONS: The frecuency of intracavitary diagnostic lesions by endoscopy in infertility patients is different than the general population Hysteroscopy is the election exam in canal anomalities. The uterus cavity endopye exploration must be recommended in the infertility study as complement of the tubarie permeability by hysterosalpingography and must be done sistematically before begining ever attempt of MAP that involve embrional transference or spermatozoons.

KEY WORDS: Hysteroscopy, infertility, study women.

\section{Introducción}

La fecundidad natural permite la obtención de un embarazo en $85 \%$ de casos después de 6 meses de relaciones sexuales regulares sin contracepción, en $80 \%$ de casos después de 1 año y en $90 \%$ después de 2 años.

Centre de Procreations Medicalement Assistees. Service de Gynecologie C., Hôpital de la Conception. Marseille-France.
Hablamos entonces de infertilidad de una pareja cuando después de 1 año de relaciones sexuales regulares (tres por semana), sin contracepción, no se obtiene un embarazo; además aproximadamente $10 \%$ de las parejas consultan por infertilidad (o sea aprox. 40-50000 parejas por ano); es entonces el examen de la pareja infértil un problema frecuente en la práctica médica. La exploración de la pareja debe hacerse conjuntamente; ya que $30 \%$ de las causas son de origen masculino, $30 \%$ de origen feme- 
nino, y $40 \%$ de la pareja misma. Dentro de las causas de origen femenino, la evaluación del factor cervical y endocavitario es fundamental; es por esto que en los últimos años los medios de exploración de la cavidad se han desarrollado y perfeccionado; siendo la endoscopia uterina la que actualmente ha alcanzado mayores logros. Como producto de este perfeccionamiento, el lugar de la histeroscopia tanto diagnóstica como operatoria, en el contexto de la infertilidad, se hace cada día mas interesante e indispensable.

\section{Materiales y métodos}

\section{Muestra}

Entre enero de 1993 y julio 1996, 184 pacientes con transtornos de la reproducción fueron explorados por histeroscopia (histerocopio flexible, de fibra óptica de $3,5 \mathrm{~mm}$ y $2,5 \mathrm{~mm}$ ) después de histerosalpingografía en el contexto del estudio de su infertilidad. Fueron descartadas 25 por información incompleta; y 13 historias clínicas no se encontraron. El estudio se realizó en el CENTRE DE PROCREATIONS MEDICALEMENT ASSISTEES. DE L'HÔPITAL DE LA CONCEPTION. MARSEILLEFRANCE.

\section{Metodología}

Estudio retrospectivo, descriptivo sobre 146 historias clínicas de pacientes que consultaron por problemas de infertilidad, a quienes se practicó una histeroscopia diagnóstica dentro del estudio de la infertilidad.

\section{Variables}

-Resultado de la histerosalpingografia,-Resultado de la histeroscopia, -Intervalo de tiempo (meses) entre histerosalpingografia (HSG) e histeroscopia (HSC), -Comparación entre el resultado a HSG y HSC (concordancia) -Antecedente de Tipo de PMA realizado y de numero de intentos. -Variables descriptivas.

Con relación a la concordancia observada entre los resultados de la HSG y de la HSC, algunos autores han publicado sus observaciones desde 1956; los resultados de nuestra serie confirman aproximadamente esta misma relación en el campo de la infertilidad. (Cuadro No. 2).

\begin{tabular}{|c|c|c|}
\hline \multicolumn{3}{|c|}{$\begin{array}{l}\text { Cuadro } 1 \\
\text { FRECUENCIA DE LAS DIFERENTES ANOMALIAS } \\
\text { ENCONTRADAS A LA HISTEROSCOPIA EN PACIENTES } \\
\text { INFERTILES Y COMPARACION CON LA POBLACION } \\
\text { GENERAL }\end{array}$} \\
\hline ANOMALIA & $\begin{array}{l}\text { PACIENTES } \\
\text { INFERTILES }\end{array}$ & $\begin{array}{l}\text { POBLACION } \\
\text { GENERAL }\end{array}$ \\
\hline $\begin{array}{l}\text { Sinequias } \\
\text { polipos } \\
\text { fibromas } \\
\text { malformaciones } \\
\text { uterinas } \\
\quad \text { - tabiques } \\
\text { anom. del canal }\end{array}$ & $\begin{array}{r}36 \% \\
11 \% \\
2-4 \%\end{array}$ & $\begin{aligned} 1,9-4,8 \% & (2)(3) \\
42,4 \% & (5) \\
-. & \end{aligned}$ \\
\hline
\end{tabular}

Cuadro 2

CONCORDANCIA ENTRE EL RESULTADO DE HSG/HSC. SEGUN ALGUNOS AUTORES.

\begin{tabular}{|lccc|}
\hline AUTOR & AÑO & $\begin{array}{l}\text { MUESTRA } \\
\text { (No.pctes) }\end{array}$ & CONCORDANCIA \\
\hline Norment & 1956 & 50 & $60 \%$ \\
Englund & 1957 & 21 & $52 \%$ \\
Endstroem & 1970 & 30 & $53 \%$ \\
Porto & 1974 & 130 & $70 \%$ \\
Parent & 1978 & 71 & $75 \%$ \\
Hamou & 1983 & 142 & $61 \%$ \\
Blanc & 1992 & 220 & $65 \%$ \\
Nuestra serie & 1996 & 146 (en infertilidad) & $47 \%$ \\
\hline
\end{tabular}

Al examinar esta tabla se debe tener en cuenta la evolución en la tecnología de los instrumentos desde 1956, $y$ en la cantidad de pacientes que conformaban estas muestras. Además pudimos evidenciar también, que si el intervalo de tiempo entre los dos exámenes aumenta, la concordancia disminuye aun más. (tabla 4., gráfica 4). Cuando tomamos a parte solo el grupo de pacientes con resultado anormal a la HSC., observamos que a mayor edad, mayor posibilidad de tener anomalías; alcanzando casi un $90 \%$ cuando las pacientes superan los 40 años.

\section{Resultados}

Tabla 1

FRECUENCIA DE ANOMALIAS EN HSG.

\begin{tabular}{|c|c|c|}
\hline RESULTADO HSG. & $\begin{array}{l}\text { No. DE } \\
\text { PACIENTES }\end{array}$ & $\%$ \\
\hline HSG. normal (0) & 26 & $18 \%$ \\
\hline Pólipo (1) & 21 & $14 \%$ \\
\hline Fibroma (2) & 6 & $4 \%$ \\
\hline Sinequia simple (3) & 15 & $10 \%$ \\
\hline Sineq. compleja (4) & 3 & $2 \%$ \\
\hline Malformación (5) & 11 & $8 \%$ \\
\hline Anom. del canal (6) & 0 & $0 \%$ \\
\hline Anomalía de la mucosa (7) & 5 & $3 \%$ \\
\hline Anom. de perfusión tubárica (8) & 37 & $25 \%$ \\
\hline $1+3(9)$ & 5 & $3 \%$ \\
\hline $1+8(10)$ & 5 & $3 \%$ \\
\hline $1+5(11)$ & 2 & $1 \%$ \\
\hline $1+2(12)$ & 1 & $1 \%$ \\
\hline $1+7(13)$ & 0 & $0 \%$ \\
\hline $2+8(14)$ & 1 & $1 \%$ \\
\hline $2+3(15)$ & 0 & $0 \%$ \\
\hline $3+5(16)$ & 3 & $2 \%$ \\
\hline $3+8(17)$ & 2 & $1 \%$ \\
\hline $4+5(18)$ & 0 & $0 \%$ \\
\hline $4+6(19)$ & 0 & $0 \%$ \\
\hline $5+7(20)$ & 1 & $1 \%$ \\
\hline $5+8(21)$ & 2 & $1 \%$ \\
\hline $6+7(22)$ & 0 & $0 \%$ \\
\hline $1+7+5(23)$ & 0 & $0 \%$ \\
\hline $5+6+7(24)$ & 0 & $0 \%$ \\
\hline $3+6+7(25)$ & 0 & $0 \%$ \\
\hline TOTAL & 146 & $100 \%$ \\
\hline
\end{tabular}


Gráfico 1

DISTRIBUCION DE ANOMALIAS A LA HSG.

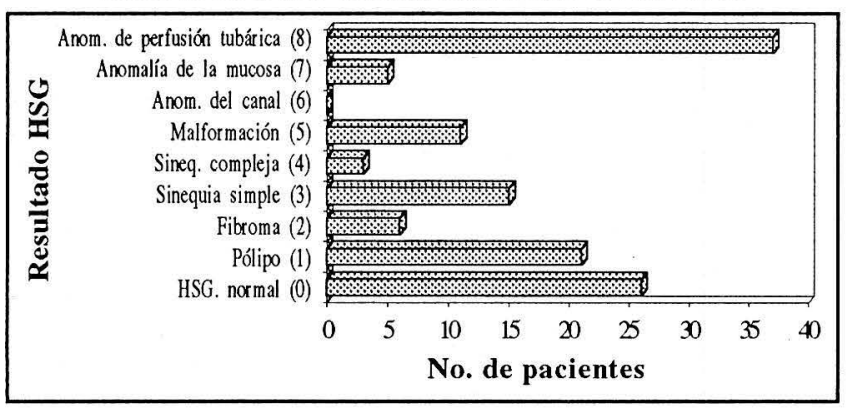

Tabla 2

RESULTADO AL EXAMEN HISTEROSCOPICO

\begin{tabular}{|lrl|}
\hline RESULTADO HSC & $\mathbf{N}^{\circ}$ PACIENTES & $\%$ \\
\hline HSC. Normal (0) & 35 & $24 \%$ \\
Pólipo (1) & 16 & $11 \%$ \\
Fibroma (2) & 2 & $1 \%$ \\
Sinequia simple (3) & 35 & $24 \%$ \\
Sinequia compleja (4) & 5 & $3 \%$ \\
Malformaciones (5) & 14 & $10 \%$ \\
Anom. del canal.(6) & 11 & $8 \%$ \\
Anom de la mucosa (7) & 6 & $4 \%$ \\
Otras (8) & 1 & $1 \%$ \\
$1+3(9)$ & 5 & $3 \%$ \\
$1+8(10)$ & 1 & $1 \%$ \\
$1+5(11)$ & 1 & $1 \%$ \\
$1+2(12)$ & 0 & $0 \%$ \\
$1+7(13)$ & 1 & $1 \%$ \\
$2+8(14)$ & 0 & $0 \%$ \\
$2+3(15)$ & 1 & $1 \%$ \\
$3+5(16)$ & 4 & $3 \%$ \\
$3+8(17)$ & 0 & $0 \%$ \\
$4+5(18)$ & 1 & $1 \%$ \\
$4+6(19)$ & 1 & $1 \%$ \\
$5+7(20)$ & 1 & $1 \%$ \\
$5+8(21)$ & 0 & $0 \%$ \\
$6+7(22)$ & 2 & $1 \%$ \\
$1+7+5(23)$ & 1 & $1 \%$ \\
$5+6+4(24)$ & 1 & $1 \%$ \\
$(3+6+7) 25$ & 1 & $1 \%$ \\
TOTAL & $\mathbf{1 4 6}$ & $\mathbf{1 0 0 \%}$ \\
\hline
\end{tabular}

Gráfico 2

DISTRIBUCION SEGUN RESULTADO HISTEROSCOPICO

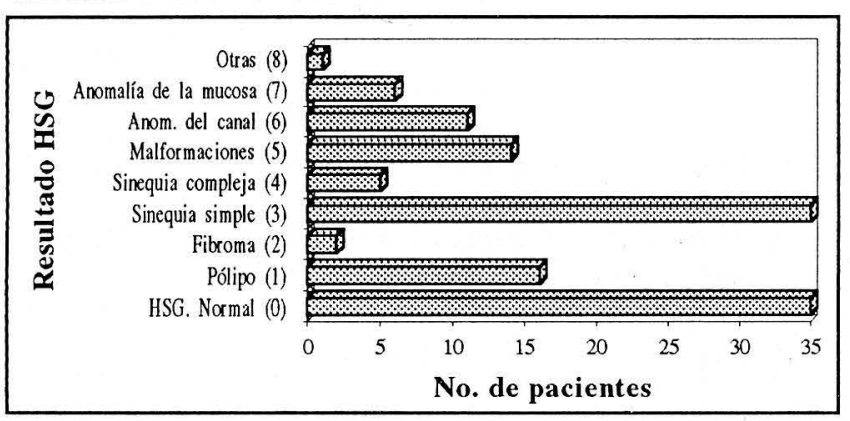

Tabla 3

COMPARACION ENTRE EL RESULTADO A LA HISTEROSALPINGOGRAFIA E HISTEROSCOPIA

\begin{tabular}{|lcl|}
\hline COMPARACION & $\begin{array}{c}\mathbf{N}^{\circ} \text { DE } \\
\text { HSG - HSC. }\end{array}$ & PACIENTES \\
\hline Concordancia & 68 & \\
Discordancia & 78 & 47 \\
Total & $\mathbf{1 4 6}$ & 53 \\
\hline
\end{tabular}

Gráfico 3

DISTRIBUCION DE LA COMPARACION ENTRE HSG Y HSC

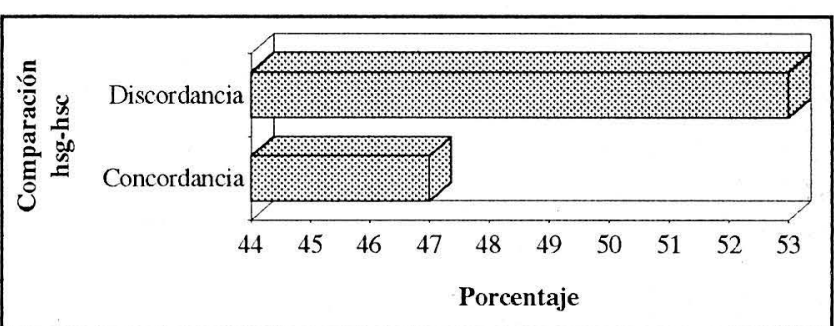

En el contexto del estudio de la infertilidad la serie muestra una concordancia del $47 \%$ entre el resultado de la HSG y HSC.

Tabla 4

DISTRIBUCION DE LA CONCORDANCIA EN FUNCION DEL TIEMPO

\begin{tabular}{|lcc|}
\hline INTERVALO (mes) & $\begin{array}{c}\mathbf{N}^{\circ} \text { DE } \\
\text { CONCORDANCIAS }\end{array}$ & $\%$ \\
\hline$<6$ & 38 & 26 \\
de 7 a 12 & 11 & 8 \\
de 13 a 18 & 8 & 5 \\
de 19 a 24 & 3 & 2 \\
$>24$ & 8 & 5 \\
Total & $\mathbf{6 8}$ & $\mathbf{4 7}$ \\
\hline
\end{tabular}

\section{Gráfico 4}

DISTRIBUCION DE LA CONCORDANCIA (HSG-HSC) EN FUNCION DEL TIEMPO

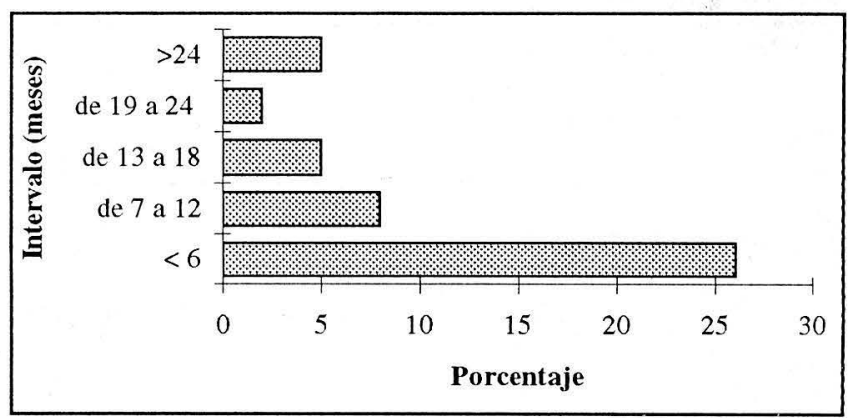

La concordancia entre la HSG y HSC es claramente descendente a medida que el intervalo de tiempo entre los exámenes aumenta. 
Tabla 5

DISTRIBUCION DE HISTEROSCOPIAS ANORMALES

SEGUN LA EDAD

\begin{tabular}{|llll|}
\hline GRUPOS & HSC. & & \\
DE EDAD & ANORMAL & TOTAL & $\%$ \\
\hline$<$ O $=20$ & 0 & 0 & 0 \\
de 21 a 29 & 16 & 29 & $55 \%$ \\
de 30 a 39 & 77 & 96 & $80 \%$ \\
$>40$ & 18 & 21 & $86 \%$ \\
Total & $\mathbf{1 1 1}$ & $\mathbf{1 4 6}$ & $\mathbf{7 6 \%}$ \\
\hline
\end{tabular}

*. Gráfico $\mathrm{N}^{\circ} 5$.

DISTRIBUCION DE HISTEROSCOPIAS ANORMALES SEGUN LA EDAD

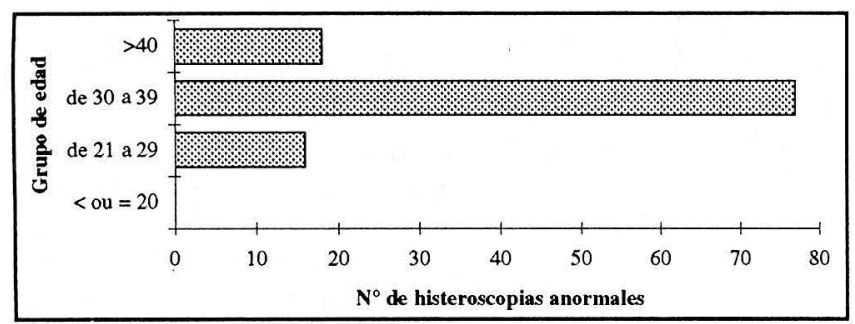

Entre las pacientes que tuvieron alguna anomalía a la histeroscopia, el grupo de edad mas afectado fue el de $>40$ años. 18 de 21 (86\%).

Tabla 6

DISTRIBUCION DE HISTEROSCOPIAS ANORMALES ENTRE LAS PACIENTES CON ANTECEDENTE DE PMA.

\begin{tabular}{|c|c|c|c|}
\hline TIPO DE PMA. & $\begin{array}{r}N^{\circ} \text { DE HSC. } \\
\text { ANORMALES }\end{array}$ & TOTAL PMA. & $\%$ \\
\hline Ninguna (1) & 50 & 78 & 64 \\
\hline Estimulación de & & & \\
\hline ovulación (2) & 14 & 16 & 88 \\
\hline Inseminación (3) & 12 & 15 & 80 \\
\hline FIV-ICSI (4) & 25 & 26 & 96 \\
\hline $4+2(5)$ & 3 & 3 & 100 \\
\hline $2+3(6)$ & 2 & 3 & 67 \\
\hline $3+4(7)$ & 5 & 5 & 100 \\
\hline Total & 111 & 146 & 76 \\
\hline
\end{tabular}

Gráfico 6

DISTRIBUCION DE HISTEROSCOPIAS ANORMALES ENTRE LAS PACIENTES CON ANTECEDENTE DE PMA.

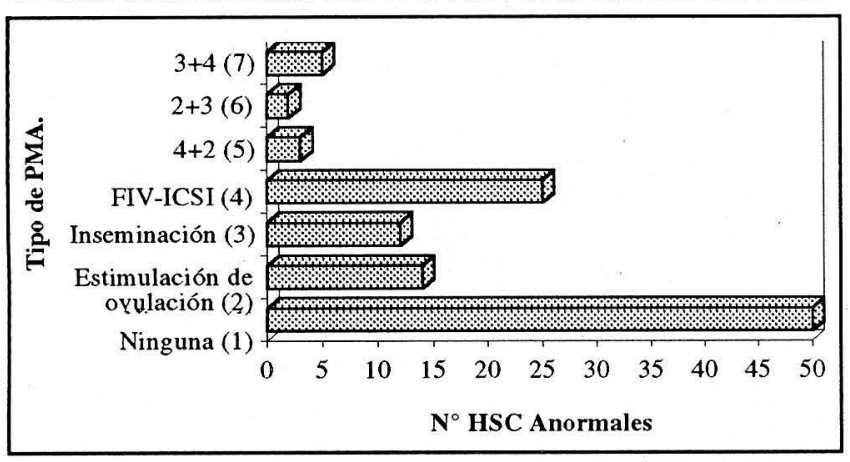

Entre las pacientes con antecedente de PMA, el grupo de FIVICSI mostró un 96\% de histeroscopias anormales, (25 de 26).
Tabla 7

RELACION DE No. DE INTENTOS FALLIDOS DE PMA E HSC ANORMAL

\begin{tabular}{|cccr|}
\hline$N^{\circ}$ INTENTOS & $\begin{array}{c}\mathbf{N}^{\circ} \text { HSC. } \\
\text { ANORMALES }\end{array}$ & $\begin{array}{c}\text { TOTAL } \\
\text { PACIENTES }\end{array}$ & $\%$ \\
\hline Ningún intento & 50 & 78 & $64 \%$ \\
1 & 5 & 5 & $100 \%$ \\
2 & 15 & 16 & $94 \%$ \\
3 & 13 & 14 & $93 \%$ \\
4 & 7 & 10 & $70 \%$ \\
5 & 3 & 4 & $75 \%$ \\
6 & 7 & 8 & $88 \%$ \\
7 & 1 & 1 & $100 \%$ \\
8 & 4 & 4 & $100 \%$ \\
9 & 3 & 3 & $100 \%$ \\
10 & 0 & 0 & $0 \%$ \\
11 & 3 & 3 & $100 \%$ \\
Total & $\mathbf{1 1 1}$ & $\mathbf{1 4 6}$ & $\mathbf{7 6 \%}$ \\
\hline
\end{tabular}

Gráfico 7

RELACION DE No. DE INTENTOS FALLIDOS DE PMA E HSC ANORMAL

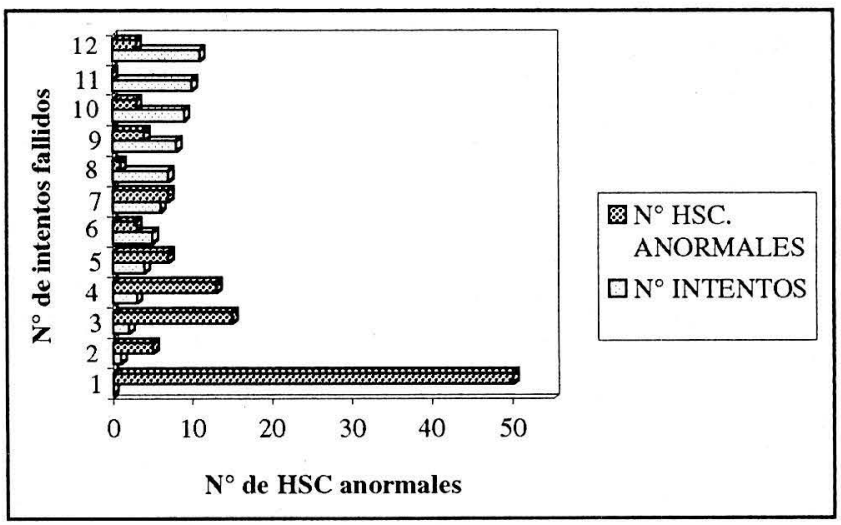

Podemos señalar que:

En el grupo de pctes sin antecedentes de PMA, 64\% tuvieron HSC. anormal.

En el grupo de pctes con antecedente de $<4$ intentos, $73 \%$ presentaron HSC anormal.

En el grupo de pctes con antecedente de $>4$ intentos, $85 \%$ mostraron HSC anormal.

\section{Discusión}

El grupo de estudio a quienes se practicó una histeroscopia diagnóstica en el contexto de su infertilidad tenía una edad promedio de 34,4 años, que corresponde a la edad promedio de mujeres que consultan por infertilidad (33-34 años).

Con relación al tipo de infertilidad no hubo diferencia significativa, ya que $49 \%$ tenían infertilidad primaria y $51 \%$ secundaria.

En cuanto al diagnóstico de las anomalías del canal endocervical pudimos evidenciar que dentro de las le- 
siones encontradas al examen histeroscópico $8 \%$ correspondían al canal endocervical, mientras que en este mismo grupo, el examen HSG no mostró ninguna alteración del endocervix. Esto puede explicarse por los cambios ejercidos sobre el canal durante examen HSG, que impiden la visualización de las alteraciones dentro del cuello (tipo de cánula, longitud introducida dentro del canal, tracción ejercida, etc.) cambios que son evitados al utilizar una fibra óptica flexible para su exploración.

Con relación a la frecuencia de anomalías encontradas al examen histeroscópico las lesiones más frecuentes fueron las sinequias: 53 casos (36\%); muy superior a la observada en la población general (10-12\%) ROGE et cols. (1). Nosotros encontramos $10 \%$ de malformaciones uterinas, de las cuales $54 \%$ correspondían a tabiques uterinos; mientras que en la población general la frecuencia de malformaciones uterinas varía de 1,9-4,8\% según ASHTON(2), SORENSEN(3), NASRI(4), de estas ultimas la frecuencia de tabiques fue $42,4 \%$ (5). La frecuencia de polipos en el grupo de mujeres que consultan por infertilidad alcanzan un $11 \%$, mientras que en la población general BLANC, BOUBLI et cols.(6) encontraron una frecuencia de $5 \%$ y una edad promedio de 48 años. CRAVELLO et cols. (7), han mostrado que la frecuencia global de fibromas varia de $4-29 \%$, mientras que en la población con transtornos de la fertilidad esta varía de 2$4 \%$ (Cuadro No. 1).

De otra parte cuando seleccionamos el grupo de pacientes con el antecedente de fertilización asistida, que implique acceso a la cavidad (FIV, ICSI, INSEMINACIONES); encontramos que 42 de 46 pacientes (93\%), tuvieron resultado histeroscópico anormal; mostrando el alto porcentaje de anomalías que se evidencian en el curso de algún intento iterativo de fertilización asistida. (Tabla 6,7-Gráficos 6,7). Lo que permite interrogarnos si en realidad hay necesidad de esperar un determinado número de fracasos para indicar una histeroscopia diagnóstica; teniendo en cuenta todo lo que representa la puesta en marcha de una fertilización asistida; o si por el contrario practicándola de entrada, evitamos al máximo la posibilidad de fracasos por anomalías endocervicales y endocavitarias.

\section{Conclusiones}

-La frecuencia de lesiones encontradas a la exploración histeroscópica en las pacientes infértiles es diferente a la de las pacientes de la población general; posiblemente como consecuencia de la selección de la serie según un problema específico (infertilidad) y una edad especifica (edad fértil). -La histeroscopia es evidentemente el examen mas preciso y adecuado para el diagnostico de las anomalías del canal endocervical. -Teniendo en cuenta que: ${ }^{*} \mathrm{La}$ concordancia entre la HSG y la HSC. es solamente del $47 \%$ y además disminuye en función del tiempo. *El número de histeroscopias anormales después de procedimientos frustros de fertilización asistida "invasiva" (FIV, ICSI, INSEMINACIONES) alcanza un $93 \%$. ${ }^{*}$ La posibilidad de tener una histeroscopia anormal en el grupo de pacientes que no ha tenido ningún intento de PMA, en el grupo con menos de 4 intentos, y en el grupo con mas de 4 intentos es de $64 \%$, $73 \%$ y $85 \%$ respectivamente. (A mayor cantidad de fracasos, mayor posibilidad de hallazgos anormales). ${ }^{*}$ Los procedimientos de PMA "invasivos" son costosos, de larga duración, y difíciles de soportar por las pacientes; *Si a esto agregamos que la HISTEROSCOPIA es un examen simple, de corta duración, ambulatorio, que no requiere anestesia, y muy económico, etc. Es lógico y pertinente recomendar la exploración endoscópica de la cavidad uterina en el estudio de la infertilidad, como complemento de la evaluación de la permeabilidad tubárica por histerosalpingografia; y de manera sistemática antes de iniciar cualquier intento de fertilización asistida que implique acceso a la cavidad (transferencia de embriones o de espermatozoides).

\section{BIBLIOGRAFIA}

1. Roge P., D'Ercole C., Cravello L., Boubli L., Blanc B. Le traitment histeroscopique des synéchies uterines. J. Gynecol. Obstet. Biol. Reprod. 1996; 25: 33-40.

2. Ashton D., Amin H., Richart RM., Neuwirth RH. The incidence of asymptomatic uterine anomalies in woman undergoing transcervical tubal sterilization. Obstet. Gynecol. 1988; 72: 28-30.

3. Sorensen S. Estmeted prevalence of müllerian anomalies. Acta Obstet. Gynecol. Scand. 1988; 67: 441-445.

4. Nasri M., Setchelll M., Chard T. Transvaginal ultrasound for diagnosis of uterine malformations. Brit. J. Obstet. Gynecol. 1990; 97: 10431045 .
5. Lavergne N. Malformations uterines. Thése pour le doctorat en médecine. Marseille 1994.

6. Lavergne N., Aristizabal J., Zarka V., Erny R., Hedon B. Uterine anomalies and in vitro fertilization: what are the results? European Journal of Obstetrics \& Gynecology and Reproductive Biology. 1996; 68: 29-34.

7. Blanc B., Boubli L. Endoscopie uterine, 2éme edition 1996.

8. Cravello L., D'ercole C., Azoulay P., Boubli L., Blanc B. Le traitement hysteroscopique des fibromes uterines. J. Gynecol. Obstet. Biol. Reprod. 1995; 24: 374-380. 